\title{
Uso de registros de temperatura máxima promedio de las estaciones meteorológicas de ETESA, para la creación de mapas de temperatura mediante el uso de programa ArcGIS
}

\section{Use of average maximum temperature records from ETESA meteorological stations, for the creation of temperature maps using the ArcGIS program}

\author{
Danna G. Villarreal ${ }^{1}$, Sergio Bernal ${ }^{2}$, Martín E. Candanedo G. $^{2 *}$ \\ ${ }^{1}$ Licenciatura en Ingeniería Ambiental- Facultad de Ingeniería Civil- Universidad Tecnológica de Panamá, ${ }^{2 *}$ Licenciatura en \\ Ingeniería Civil- Facultad de Ingeniería Civil- Universidad Tecnológica de Panamá
}

Resumen Ante el incremento del consumo energético en la República de Panamá debido a la sensación térmica en la población, nace la idea de verificar la existencia de un incremento de la temperatura con el tiempo. Bajo este objetivo, se analizan registros de temperaturas máximas de las estaciones meteorológicas de la Empresa de Transmisión Eléctrica, S.A. (ETESA) para el período comprendido entre 2010-2017. Al no existir registros para muchas zonas en el país, se tabulan los datos de temperaturas máximas por meses y se calculan los promedios por año. Estos valores sirven para la creación de mapas de temperaturas máximas en el territorio nacional utilizando el programa ArcGIS. Estos mapas confirmaron un incremento considerable de temperatura desde el año 2010.

Palabras clave ArcGIS, estación hidrometeorológica, ETESA, mapa de temperatura, sensación térmica, temperatura.

\begin{abstract}
Given the increase in energy consumption in the Republic of Panama due to the thermal sensation in the population, the idea of verifying the existence of an increase in temperature over time was born. With this objective, records of maximum temperatures of the meteorological stations of Empresa de Transmission Eléctrica, S.A. (ETESA) are analyzed for the period between 2010-2017. As there are no records for many areas in the country, maximum temperature data are tabulated by months and averages are calculated by year. These values are used to create maps of maximum temperatures in the national territory using the ArcGIS program. These maps confirmed a considerable increase in temperature since 2010.
\end{abstract}

Keywords ArcGIS, hydrometeorological station, ETESA, temperature map, thermal sensation, temperature.

* Corresponding author: martin.candanedo@utp.ac.pa

\section{Introducción}

Panamá experimenta un gran auge en el sector de la construcción. El número de permisos de construcción aumentaron $19 \%$ en los primeros cuatro meses de 2017, respecto al mismo periodo del año previo [1]. Sin duda alguna, este progreso en el sector solo indica que el número de construcciones va en ascenso. De igual manera, con el pasar de los años, las olas de calor y la sensación de fogaje en el país son cada vez más frecuentes. Estas sensaciones han alcanzado valores tan altos como 42 grados centígrados [2]. Esto puede deberse a la relación directa entre el área de construcción y de asfalto con el incremento en la sensación térmica en diferentes ciudades [3].
La Empresa de Transmisión Eléctrica de Panamá -ETESA comunica que esto puede deberse a la temporada y el clima del país. Sin embargo, también comenta que la brisa que se sentía en veranos anteriores se está debilitando [2]. Es un hecho que en la ciudad este fenómeno se magnifica con el aumento de áreas urbanizadas en la ciudad y la pérdida de vegetación. Esta situación produce un alto consumo energético, debido al uso generalizado de acondicionadores de aire [4]. Así, el consumo energético llega en el año 2017 al valor de $1650 \mathrm{~kW}$, siendo una cifra récord sin precedentes [5].

Para poder encontrar una explicación a esta observación, primero se debe determinar si en realidad los registros de temperatura que existen muestran incrementos o si es una cuestión de sensación (percepción) térmica. 


\subsection{Objetivos:}

- Determinar la existencia de un incremento de la temperatura máxima promedio a nivel nacional por medio del análisis del registro de datos de estaciones meteorológicas de ETESA.

- Desarrollar mapas de temperatura, mediante el uso del programa ArcGIS con los registros de temperatura.

\section{Antecedentes}

El incremento de las ciudades como respuesta al crecimiento de la población trae consigo el incremento de la densidad de edificios y el aumento de áreas de construcción. Este desarrollo urbano influye en la modificación de la distribución espacial de la temperatura y humedad [7]. Este cambio en la temperatura trae consigo afectaciones en la calidad de vida de la población y otros seres vivos, atribuida en muchos casos al incremento de la sensación térmica.

Es importante hacer la salvedad de que la sensación térmica es determinada por un conjunto de parámetros como lo son: la temperatura seca, la temperatura radiante media o temperatura de bulbo negro, la humedad relativa del aire y la velocidad del aire, siendo la sensación térmica un mal indicador directo de temperatura [8]. Aquí radica la importancia de medir directamente la temperatura y no la sensación térmica, ya que al medirla estamos midiendo también los factores que determinan su crecimiento.

El tener una infraestructura verde y vegetación sana, permite reducir estos riesgos hidrometeorológicos [9]. Se ha identificado una correlación entre los registros de temperatura más bajos y espacios con mayor vegetación [7]. Sin embargo, en áreas rurales esta riqueza de vegetación se ha visto comprometida por motivo de la deforestación, la ganadería y el uso de fertilizantes [10], además del incremento de construcciones.

Por parte de la naturaleza, se deben tomar en cuenta los aspectos climáticos presentes en cada región del país. Otra posible causa de este incremento de las temperaturas es el aumento de los gases de efecto invernadero o GEI. Los GEI son generados por actividades antropogénicas urbanas, como el consumo elevado de combustibles fósiles, el alto consumo de energía eléctrica y automóviles, el uso de materiales de bajo albedo y la mala geometría urbana [3].

Es de vital importancia reconocer que el incremento de temperaturas tiene otras afectaciones en el aspecto climatológico como el aumento del nivel del mar, creando erosión costera, inundaciones y afectaciones a los ecosistemas coralinos [11]. Además, cambios muy altos de temperaturas pueden tener afectaciones en la salud de las personas por la gran concentración de calor [12].

\section{Delimitación de datos}

Para la realización de esta investigación se tomó como datos los registros de temperaturas promedios y temperaturas máximas que tiene a disposición la Empresa de Transmisión Eléctrica, S.A. (ETESA) y se hizo una tabulación de estos. El período elegido para el análisis fue de 2010- 2017. Las temperaturas promedio por mes son graficadas para ver su comportamiento, mientras que las máximas son indicativas de la condición más crítica de temperatura y el principal interés de esta investigación.

\section{Metodología}

\subsection{Selección de estaciones meteorológicas}

De las once estaciones meteorológicas vigentes y con registros en el periodo 2010-2017, solamente las estaciones de Tocumen, Albrook, David, Santiago y Los Santos cuentan con registros de datos de temperaturas promedio durante todos los meses de este periodo. En la figura 1, se demuestra el formato para la tabulación de datos por parte de ETESA. Para el análisis de temperaturas máximas promedio en cada mes, se usó, además, la estación de Bocas del Toro.

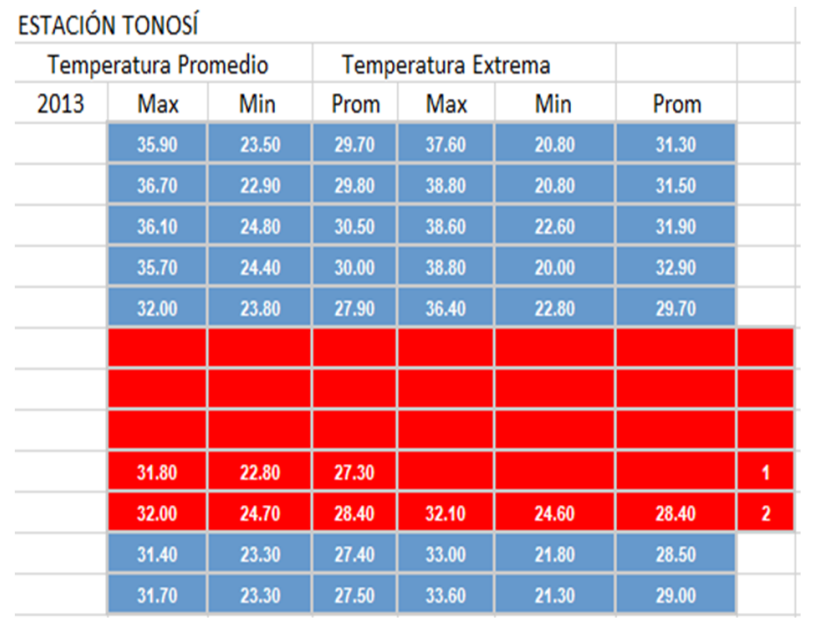

Figura 1. Durante la selección de datos, estaciones como Tonosí no contaban con suficientes datos de temperatura y por esto, no fueron analizados.

\subsection{Gráficas de temperaturas promedio y temperaturas máximas}

Con los registros obtenidos de las estaciones con las temperaturas promedio, se hicieron gráficas de barras en Excel, para poder observar cuál era su comportamiento. Se graficaron los datos de los años 2010, 2013 y 2017 obtenidos de las estaciones de Tocumen, Albrook, David, Santiago y Los Santos.

Además, con los registros de 2010-2017, se realizó una gráfica de dispersión en Excel con los datos de las temperaturas máximas para las estaciones meteorológicas de Tocumen, Albrook, David, Santiago, Los Santos y Bocas del Toro.

\subsection{Creación de mapas de temperatura con ArcGIS}

Tras obtener las coordenadas de cada una de las estaciones meteorológicas, se transformaron a proyección de coordenadas UTM (WGS 84), se subieron al programa 
ArcGIS junto con los registros de temperaturas máximas para cada estación desde 2010 a 2017. Luego, se escogió un método de interpolación apropiado para crear los diferentes mapas de temperaturas de cada año para obtener una distribución de las temperaturas máximas en estos años a nivel nacional.

\section{Resultados}

En la figura 2 se comparan las estaciones de Tocumen, Albrook, David, Santiago y Los Santos, observándose un incremento gradual de la temperatura promedio, con excepción de Tocumen y Albrook, donde se observa una pequeña disminución desde 2013 (ver figura 2). Sin embargo, cuando se observan las temperaturas máximas extremas (mayores valores de temperaturas máximas en cada mes del año) se observa que los valores son cada vez mayores.

En Tocumen, para abril 2017, se muestra una temperatura máxima de $35,9^{\circ} \mathrm{C}$, mientras que la máxima en todo el año 2013 es de $34,60^{\circ} \mathrm{C}$. Al observar la estación de Albrook, se observa una temperatura máxima de $35,5^{\circ} \mathrm{C}$ en el mes de abril, mientras que la máxima registrada en todo el 2013 fue de $35^{\circ} \mathrm{C}$. Esto quiere decir que a pesar de que las temperaturas promedio no necesariamente incrementan, cada vez se observan valores mayores de temperaturas en fechas individuales. Este decrecimiento en temperatura promedio también puede deberse al fenómeno del "El niño" [13].

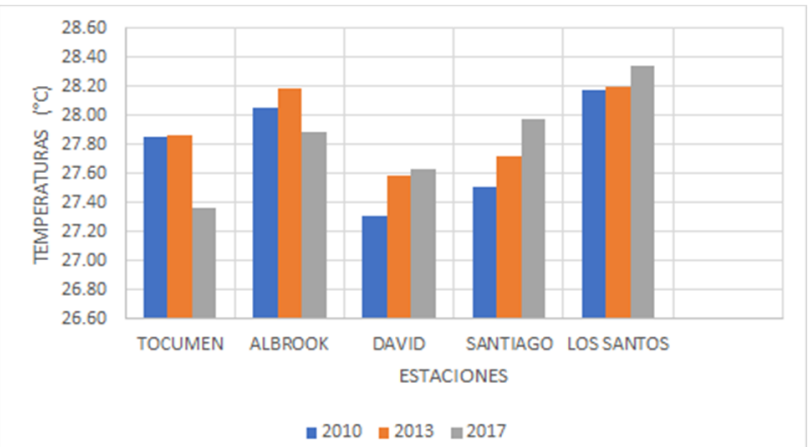

Figura 2. Gráfica de barras de registros de temperaturas promedio. Se observa un incremento de temperatura promedio en David, Santiago y los Santos. En Tocumen y Albrook hay incrementos de temperaturas máximas.

Ahora, tomando en cuenta esta información, se procede a analizar las temperaturas máximas para cada una de las estaciones con registros (incluida la estación meteorológica de Bocas del Toro). Al analizar la figura 3 , se puede concluir fácilmente que las temperaturas han ido incrementando desde 2010 hasta la fecha y que el pico de promedios de temperatura máximo se ve en 2015. Los comportamientos en cinco de las seis estaciones meteorológicas son bastante similares (Tocumen, Albrook, David, Santiago, Los Santos). Los registros en Bocas del Toro, al igual que en las otras estaciones muestran un incremento de las temperaturas promedio máximas. Sin embargo, el comportamiento no es tan parecido al que muestran las otras líneas.

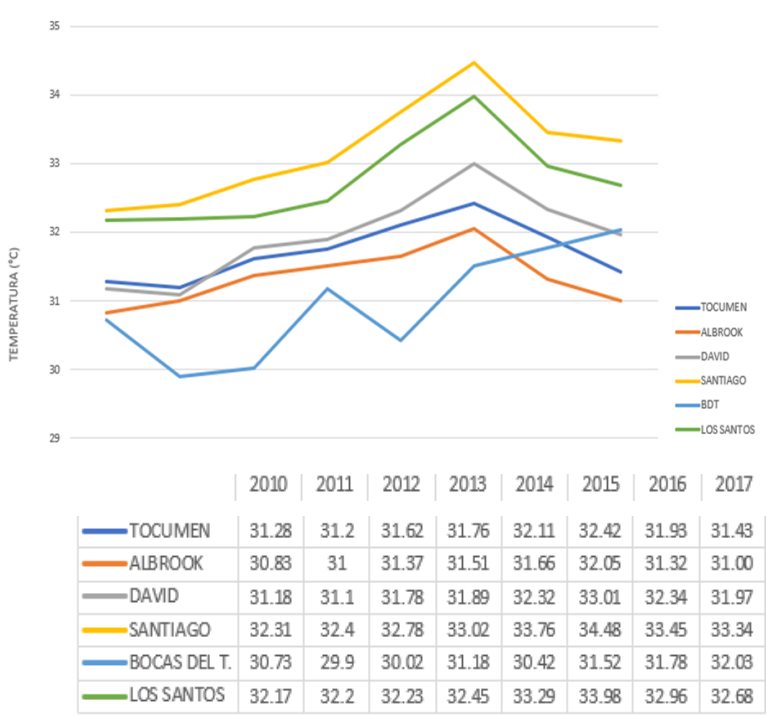

Figura 3. Gráfica de dispersión de temperaturas máximas promedio por año (2010-2017). Nótese el incremento de temperatura máxima en todas las estaciones desde 2010.

Para la selección del método de interpolación, se han utilizado los métodos de Kringin, Lineal y de ponderación por distancia inversa (IDW, Inverse Distance Weight). Al observar la figura 4, el comportamiento de las isotermas, se escoge la que mejor representa la distribución real de temperaturas en el país, haciendo referencia a los datos de ETESA. Cabe destacar que el método de IDW y Krigin presentan cierta similitud mientras el método lineal dispersa los valores del rango de comportamiento de los datos. El método de IDW es el más apropiado, por su mayor representatividad con respecto al comportamiento descrito en el registro de temperatura.

Una vez seleccionado el método de interpolación, se procede a generar los mapas con los registros de temperatura promedio máxima anual para el período 2010 al 2017 (ver figuras 5-8). Se observa como el espectro de temperatura se amplifica en los algunos sitios adyacentes al área de influencia de las estaciones de medición, mostrando un aumento de temperatura desde el año 2010 hasta el 2014 y una disminución considerable en los años restantes (ver figura 3 ).

Se puede identificar el mayor patrón de aumento de temperatura a nivel nacional en el año 2012, siendo esto un factor a considerar en la sensación térmica de dicho año. Un posible efecto consecuente para el año 2013, puede ser la crisis en los niveles de agua de embalses de hidroeléctricas, que condujo al desarrollo de un plan de emergencia de ahorro energético [14]. 
Candanedo (et al): Uso de registros de temperatura máxima promedio de las estaciones meteorológicas de ETESA, para la creación de mapas de temperatura mediante el uso de programa ArcGIS

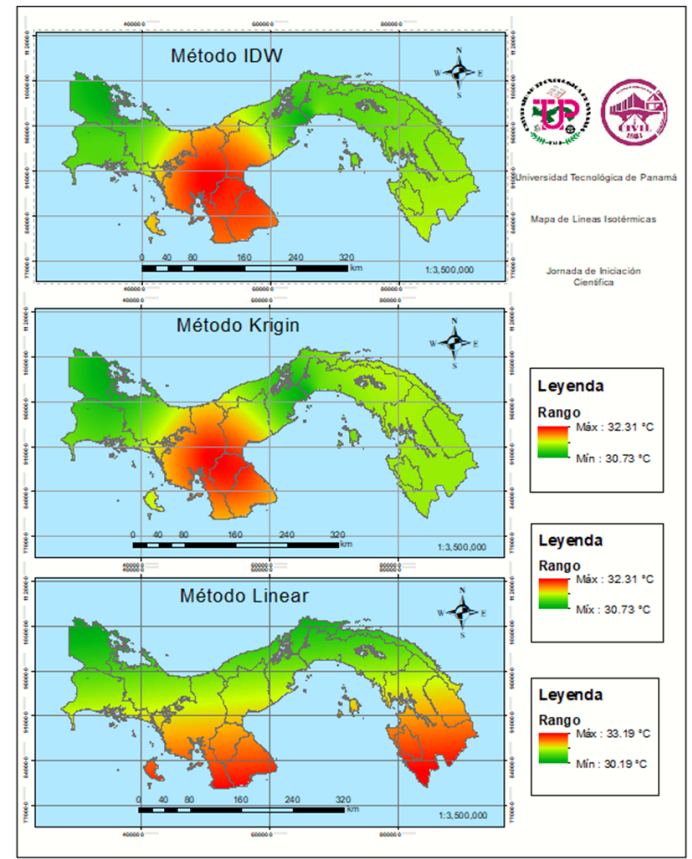

Figura 4. Análisis cartográfico del método de interpolación para registros de temperatura máxima promedio del año 2010.
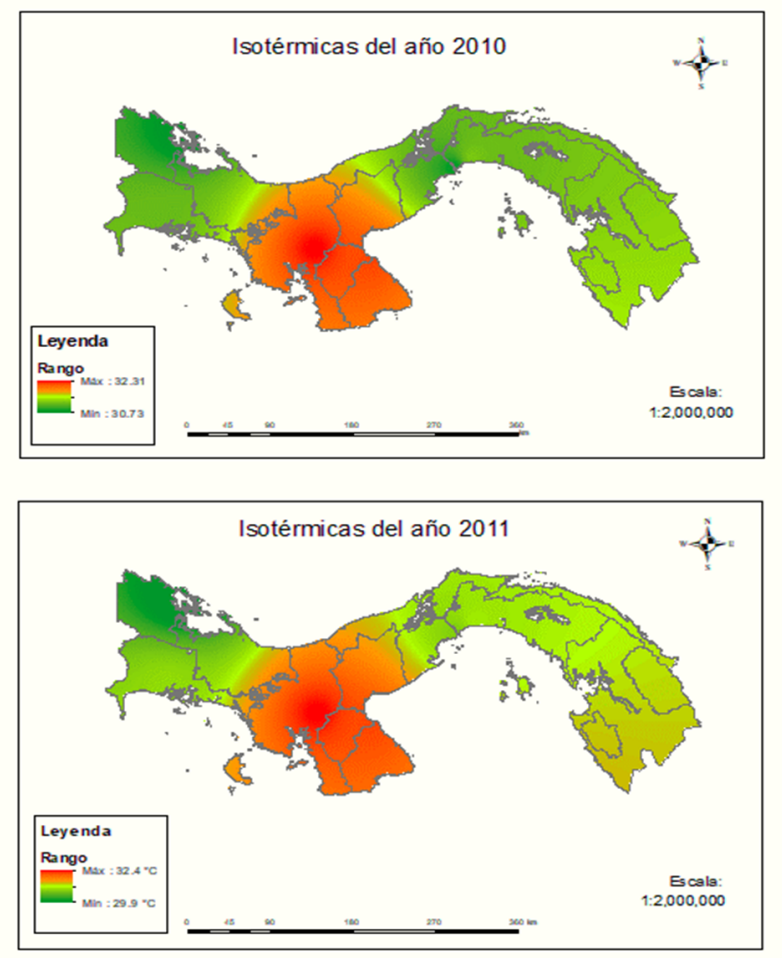

Figura 5. Análisis multitemporal comparativo de las cartográfico temperaturas máximas promedio por año (2010-2011).
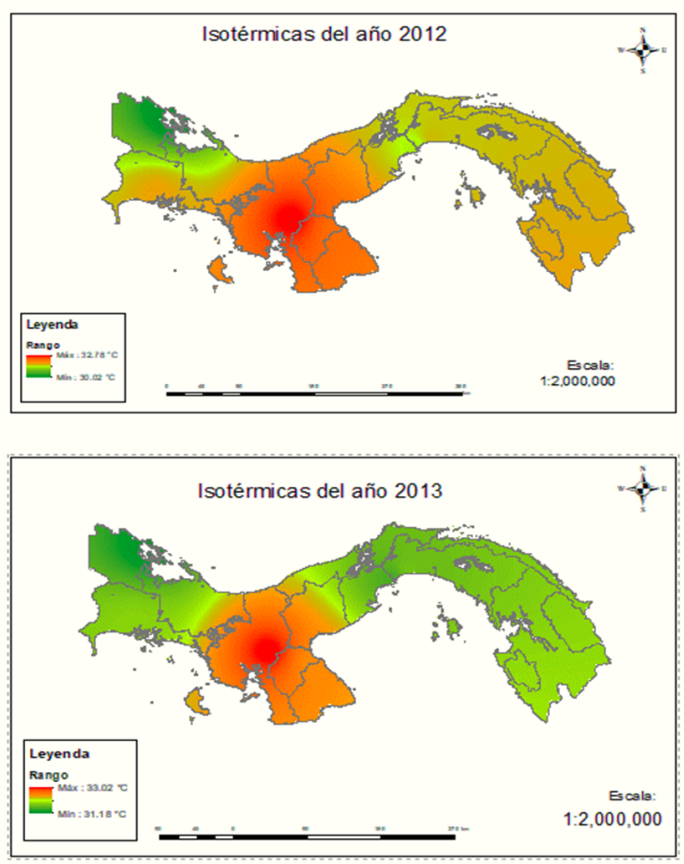

Figura 6. Análisis multitemporal comparativo de las cartográfico temperaturas máximas promedio por año (2012-2013).
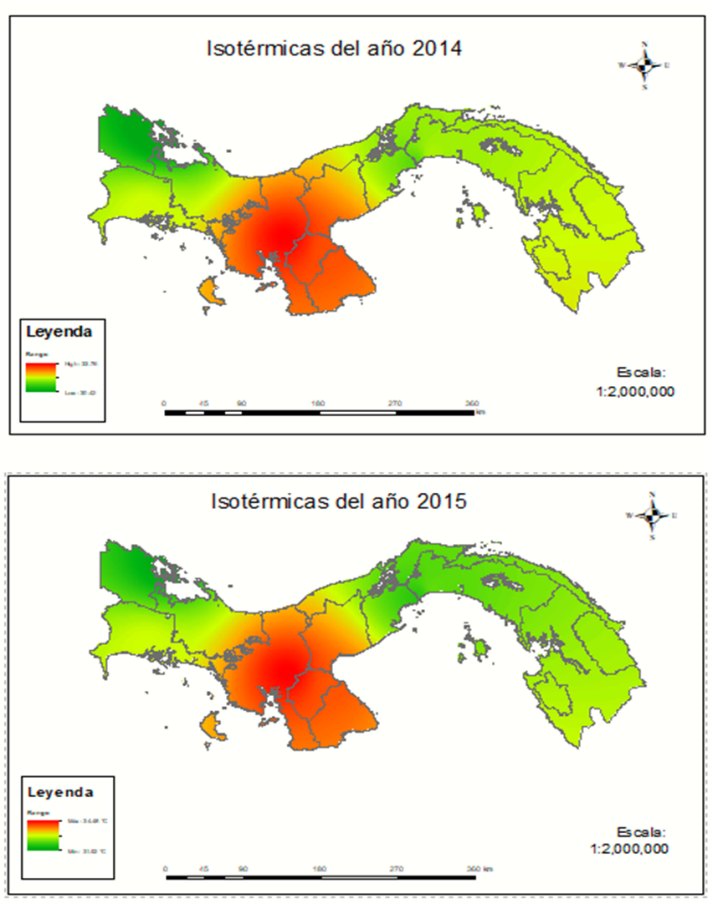

Figura 7. Análisis multitemporal comparativo de las cartográfico temperaturas máximas promedio por año (2014-2015). 

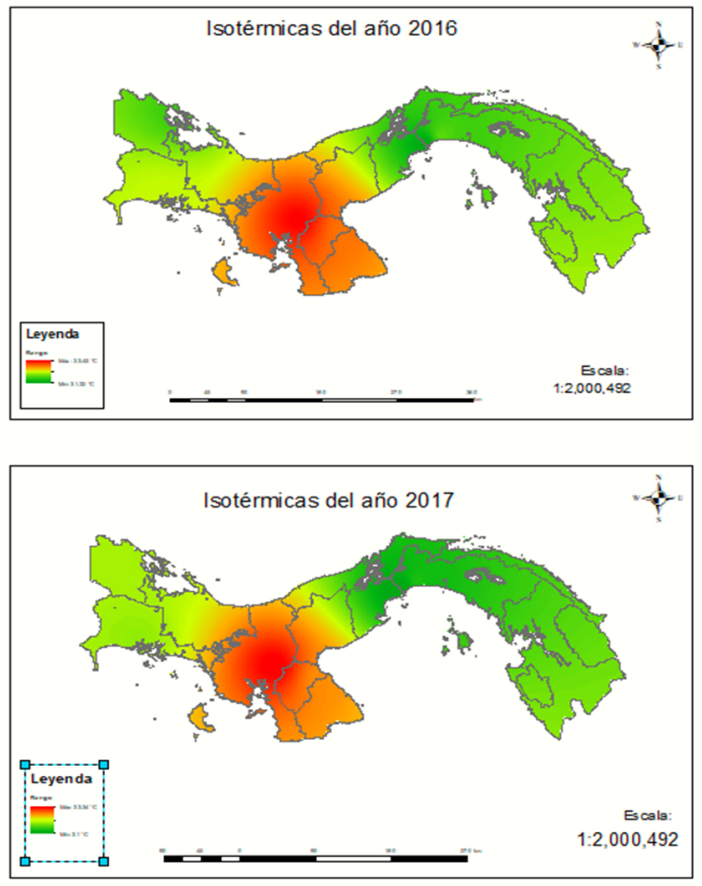

Figura 8. Análisis multitemporal comparativo de las cartográfico temperaturas máximas promedio por año (2016-2017).

\section{Conclusiones y recomendaciones}

Gracias al tabulado y selección apropiada de los datos a utilizar en esta investigación, se puede concluir que existe un incremento en la temperatura máxima promedio registrada en las estaciones meteorológicas, lo que posiblemente explica además, el incremento en la sensación térmica y el mayor consumo energético.

El aumento de temperatura posiblemente esté vinculado a otros factores, como el desarrollo urbano, el aumento de construcciones, las alteraciones en el clima en las distintas áreas de la República de Panamá, donde puede traer como consecuencia la reducción de zonas verdes por la deforestación y la ganadería.

Además, el uso del programa ArcGIS permitió la confección de mapas de temperatura para poder visualizar de una manera más comprensible el comportamiento de las temperaturas. Con los registros ya existentes de temperaturas máximas promedio de las estaciones meteorológicas incompletas se pudo elegir el método indicado para encontrar el mapa de temperatura mejor ajustado a la realidad.

Las estaciones de Tocumen, Albrook, Santiago, David y Los Santos exhibieron un comportamiento parecido cuando se analizó la variación de la temperatura máxima promedio con respecto al tiempo. Las temperaturas promedio también presentaron aumento en Los Santos, Santiago y David. En Tocumen y Albrook los valores de temperaturas extremas máximas también presentaron un incremento en los años 2010 y 2013. A pesar de que las temperaturas promedio no necesariamente incrementan, cada vez se observan valores mayores de temperaturas en fechas individuales.

Se recomienda la implementación de un mejor sistema de registro de datos por parte de ETESA, ya que la cantidad de datos faltantes hace que el tabulado y análisis de resultados sea complejo y desalentador. Solamente existieron cinco estaciones meteorológicas con los registros 2010-2017 de temperaturas promedio y seis con los registros de temperatura promedio máxima en esa misma cantidad de tiempo.

Se propone la implementación de medidas para disminuir y mantener la temperatura en rangos aceptables en Panamá como la reforestación, la implementación de ganadería y agricultura sostenible, disminuir el consumo de energía eléctrica y mejorar sistemas de transporte público para incentivar el uso de este.

\section{AGRADECIMIENTO}

Al ingeniero Daniel Cachafeiro, por habernos inspirado a realizar este artículo. Al profesor Raúl Elizondo, por su apoyo y su tiempo para solucionar nuestras dudas y ayudarnos en la realización de esta investigación y por su gran ayuda durante el manejo de datos en el programa ArcGIS.

\section{REFERENCIAS}

[1] M. González. (3 de Abril de 2017). Ola de calor en Panamá alcanza los 42 grados centígrados. Panamá América.

[2] M. Rodríguez. (4 de Julio de 2017). Permisos de construcción crecen 19\% hasta abril 2017. La Estrella de Panamá.

[3] United States Environmental Protection Agency. (2008). Obtenido de Heat Island Compendium: https://www.epa.gov/heat-islands/heat-island-compendium

[4] A. Lawrence, J.r., K Berko, \& P. du Pont. (1996). Air Conditioning in the Tropics: Cool Comfort or Cultural Conditioning? Delaware: Center for Energy and Environmental Policy.

[5] R. Pérez. (15 de Abril de 2017). Se registra consumo récord de energía en Panamá, en meses calurosos. El capital financiero.

[6] P. Pérez. (22 de Marzo de 2018). Etesa proyecta un aumento en el consumo de energía entre abril y mayo de 2018. El capital financiero. [Online]. Available: shorturl.at/ehHMT

[7] F. Ferrelli, M. Bustos, M. Piccolo, "Modificación en la distribución espacial de temperatura y la humedad relativa como resultado del crecimiento urbano" Revista de Climatología, vol. 16: 51-61, Abril 2016. [Online]. Available: shorturl.at/ezDR6.

[8] E. Urriola, "“Índice de Confort, Sensación Térmica e Impacto de Olas de Calor en las Personas"," 2007. [Online]. Available: http://www.hidromet.com.pa/sensacion_termica.php.

[9] Y. Ochoa, L. Ojela, "Conservación de vegetación para reducir riesgos hidrometeorológicos en la metrópoli fronteriza" Estudios Fronterizos, vol. 18 no. 35, Abril 2017. [Online]. Available: https://doi.org/10.21670/ref.2017.35.a03.

[10] U. EPA, "Heat Island Effect," 28 february 2014. [Online]. Available: www.epa.gov/heat-islands.

[11] C. M. Kauffman and J. M. Moran, Our Changing Climate: Introduction to climate science, Boston: American Meterorological Society, 2014. 
Candanedo (et al): Uso de registros de temperatura máxima promedio de las estaciones meteorológicas de ETESA, para la creación de mapas de temperatura mediante el uso de programa ArcGIS

[12] G. Luber and M. Micheal, "Climate Change and Extreme heat events," American Journal of Preventive Medicine, vol. 12, pp. 8773-8789, 2008

[13] ETESA. Fenómeno El Niño, [Online]. Available: http://www.hidromet.com.pa/nino_nina.php.

[14] M. Vega, "Supermecados, bares y discotecas también deben acogerse al ahorro energético", La Prensa, 07 mayo 2013. 